\title{
Coherent amplitude modulation of electron-beam-driven Langmuir waves
}

\author{
K. Baumgärtel \\ Leibniz-Institut für Astrophysik Potsdam, An der Sternwarte 16, 14482 Potsdam, Germany \\ Correspondence to: K. Baumgärtel (uk.baumgaertel@t-online.de)
}

Received: 19 December 2012 - Revised: 17 March 2013 - Accepted: 20 March 2013 - Published: 10 April 2013

\begin{abstract}
A linear approach to the phenomenon of irregular amplitude modulation of beam-driven Langmuir waves, developed in a previous paper, is extended to explain periodic modulation as well. It comes about by beating of the fastest growing mode of the instability with beam-aligned plasma oscillations. They are naturally generated in a uniform domain of beam-plasma interaction prior to the onset of the instability. Particle-in-cell (PIC) simulations support the results of the linear analysis.
\end{abstract}

Keywords. Space plasma physics (Waves and instabilities)

\section{Introduction}

Amplitude-modulated electron-beam-driven Langmuir waves have been observed by many spacecraft under different space plasma conditions during the last three decades (Gurnett et al., 1993; Hospodarsky et al., 1994; Kellog et al., 1996; Kojima et al., 1997; Pottelette et al., 1999; Sigsbee et al., 2010). The observed Langmuir envelopes show a considerable variety. Most of them are highly structured and have an irregular, random time variation with wave localizations of temporal scales ranging from $100 \omega_{\mathrm{pe}}^{-1}$ to $1000 \omega_{\mathrm{pe}}^{-1}\left(\omega_{\mathrm{pe}}\right.$ electron plasma frequency). Occasionally, however, the modulation appears coherently and the wave packets repeat their shape almost periodically in time. During such intervals the power spectrum of the longitudinal electric field exhibits a twin peak structure near $\omega_{\text {pe }}$.

The irregular Langmuir envelope modulation has been clearly reproduced in a series of particle-in-cell (PIC) simulations of electron-beam-plasma interaction (Omura et al., 1996; Muschietti et al., 1996; Matsukiyo et al., 2004; Usui et al., 2005) and in electrostatic Vlasov simulations (Umeda, 2006; Silin et al., 2007; Daldorff et al., 2011; Sauer and
Sydora, 2012), but the simulations do not reveal the physical nature of the phenomenon. Coherent modulation and the associated double peak have as yet not been explained, neither theoretically nor in terms of numerical simulations.

In a previous paper (Baumgärtel, 2013, hereafter referred to as B1), a simple linear model of the gentle beam-plasma instability has been developed that turned out to be capable of explaining primary characteristics of the irregular type of the modulation. The model, however, fails in describing coherent modulation.

The present brief report is motivated by the aspect to remove this limitation and to complete the linear model to become applicable to the periodic Langmuir envelope modulation as well.

In Sect. 2 the beam-plasma system is examined in terms of a simple fluid description, and a linear mechanism is pointed out, which may be responsible for the change from chaotic to periodic modulation. In Sect. 3 this mechanism is combined with the field calculation in B1, and its effect on the modulation is demonstrated. Section 4 presents PIC simulations that provide the chance to check the linear model.

\section{Beam-aligned plasma oscillations}

We start with a look at the initial phase of the weak beamplasma instability from a fluid point of view. Consider a situation where a uniform electron background at rest of density $n_{\mathrm{e}}$ is penetrated by a thin electron beam of density $n_{\mathrm{b}}$ and velocity $V_{\mathrm{b}}$. In absence of spatial gradients (i.e., $n_{\mathrm{e}}$ and $n_{\mathrm{b}}$ constant), the dynamics of the two separate electron continua is described by the simple system 
$\frac{d v_{\mathrm{e}}}{d t}=-\frac{e}{m_{\mathrm{e}}} E$

$\frac{d v_{\mathrm{b}}}{d t}=-\frac{e}{m_{\mathrm{e}}} E$

$\frac{d E}{d t}=\frac{e}{\epsilon_{0}}\left(n_{\mathrm{e}} v_{\mathrm{e}}+n_{\mathrm{b}} v_{\mathrm{b}}\right)$.

After eliminating $v_{\mathrm{e}}$ and $v_{\mathrm{b}}$, this system shrinks to the equation

$$
\frac{d^{2} E}{d t^{2}}-\omega_{\mathrm{pe}}^{2} E=0
$$

for plasma oscillations where $\omega_{\mathrm{pe}}$ is built from the total electron density $n_{0}=n_{\mathrm{e}}+n_{\mathrm{b}}$. This does not imply, however, that all electrons need to oscillate coherently. If we impose the initial condition $E(0)=0, v_{\mathrm{e}}(0)=0, v_{\mathrm{b}}(0)=V_{\mathrm{b}}$, we find after some algebra the solution

$$
\begin{aligned}
E(t) & =-\frac{m_{\mathrm{e}}}{e} \frac{n_{\mathrm{b}}}{n_{0}} V_{\mathrm{b}} \omega_{\mathrm{pe}} \sin \omega_{\mathrm{pe}} t \\
v_{\mathrm{e}}(t) & =-\frac{n_{\mathrm{b}}}{n_{0}} V_{\mathrm{b}}\left(\cos \omega_{\mathrm{pe}} t-1\right) \\
v_{\mathrm{b}}(t) & =V_{\mathrm{b}}+v_{\mathrm{e}}(t) .
\end{aligned}
$$

That is, the system oscillates with the plasma frequency around the initial state with an amplitude controlled by the product of beam density and beam velocity. This solution suggests that an electron beam, present in a uniform plasma, is associated with plasma oscillations of amplitude

$$
E_{0}=\frac{m_{\mathrm{e}}}{e} \omega_{\text {pe }} v_{\text {the }} \frac{n_{\mathrm{b}}}{n_{0}} \frac{V_{\mathrm{b}}}{v_{\text {the }}} .
$$

The spatially uniform field of this beam-aligned plasma oscillations was pointed to by Omura et al. (1996). These authors, however, considered it as unrealistic solution of the system without physical meaning and cancelled it from their analysis.

The electric field in Eq. (8) is expressed in units of $m_{\mathrm{e}} / e v_{\text {the }} \omega_{\text {pe }}$. To get a value in $\mathrm{Vm}^{-1}$, we refer to a plasma with $n_{0}=4 \mathrm{~cm}^{-3}, T_{\mathrm{e}}=10 \mathrm{eV}$, which gives $m_{\mathrm{e}} / e v_{\text {the }} \omega_{\mathrm{pe}} \approx$ $1.8 \mathrm{~V} \mathrm{~m}^{-1}$. Combined with the beam parameters used in B1 $\left(n_{\mathrm{b}} / n_{0}=0.05, V_{\mathrm{b}} / v_{\text {the }}=7\right)$, we get $E_{0} \approx 630 \mathrm{mV} \mathrm{m}^{-1}$. Note that the oscillation occurs before the beam-plasma instability sets in. Because of its fluid character, this phenomenon is not restricted to the initial phase but persists even in the later period of the instability after saturation when the beam has entered the plateau stage. Both density and bulk velocity of the beam are not affected by the flattening of the beam velocity distribution; i.e., the condition for the oscillation is maintained for a longer time. As a consequence, the plasma oscillation $\left(k=0, \omega=\omega_{\text {pe }}\right)$ may interfere with waves excited by the instability. According to kinetic dispersion theory, the frequency of the wave with the greatest temporal growth is generally slightly downshifted from $\omega_{\text {pe }}$.
This downshift varies relatively weakly with the beam velocity but is sensitive to variation of the beam density. For beam densities lower than $n_{\mathrm{b}} / n_{0}=0.01$, there is practically no downshift. In cases where the frequency of the dominating mode is separated from $\omega_{\text {pe }}$ and its saturated amplitude is comparable to the field strength of the plasma oscillations, one may expect a beating scenario of two waves with a small difference in frequency. The beating process results in a periodic time variation of the field envelope at a certain location associated with a double peak in the power spectrum. To establish such a scheme, a relatively dense beam is required to be involved. With decreasing beam density the two peaks tend to merge.

It should be mentioned that the field strength $E_{0}$ in Eq. (8) results from an idealized one-dimensional situation of spatially uniform ensembles of background and beam electrons. In a real space plasma, embedded in a magnetic field, such a condition would only be approximately realized. That is, the field strength in Eq. (8) should rather be considered as an upper limit of what may be reached.

\section{An example for the appearance of a double peak}

The formalism developed in B1 can easily be extended to include the plasma oscillations. One needs only to add the corresponding field to the right-hand side of Eq. (5) in B1, which then reads

$$
\begin{aligned}
E(x, t)=\frac{1}{2 \pi} \int_{-\infty}^{\infty} E(k) \exp [ & -i \omega(k) t+i k x] d k \\
& +E_{0} \exp \left[i\left(\omega_{\mathrm{pe}} t+\varphi\right)\right]
\end{aligned}
$$

with $\varphi$ an arbitrary phase. The spectral function $E(k)$ was defined in Eq. (4) of B1 as

$$
E(k)=\left[\begin{array}{lc}
E_{\mathrm{r}}(k) \exp \left[\gamma(k) t_{\mathrm{g}}\right] & \text { for } \gamma(k)>0 \\
E_{\mathrm{r}}(k) & \text { elsewhere. }
\end{array}\right.
$$

Here, $\gamma(k)$ is the growth rate of the instability predicted by the dispersion relation (see Fig. 1 of B1), $t_{\mathrm{g}}$ the growth time, and $E_{\mathrm{r}}(k)$ the wavenumber spectrum of the thermal longitudinal high-frequency noise. $\omega(k)$ is the dispersion law for the Maxwell-plateau distribution shown in Fig. 2 of B1. The integration interval in Eq. (9) naturally shrinks to positive $k$, because the beam amplifies only Langmuir waves propagating in its own flow direction.

Since the additional field is uniform, the spatial structure of $E(x, t)$ is not affected, except that the average at a fixed time is given by the momentary value of $E_{0} \exp \left[i\left(\omega_{\mathrm{pe}} t+\varphi\right)\right]$ rather than zero. The effect of the beam-aligned plasma oscillation on the temporal modulation pattern is illustrated in Fig. 1. A higher beam velocity $\left(V_{\mathrm{b}} / v_{\text {the }}=15\right)$ has been used here, which creates a stronger amplitude $E_{0}=1.3 \mathrm{~V} \mathrm{~m}^{-1}$ of the plasma oscillations. This field strength is comparable to the peak values of the field represented by the $k$-integral. As 

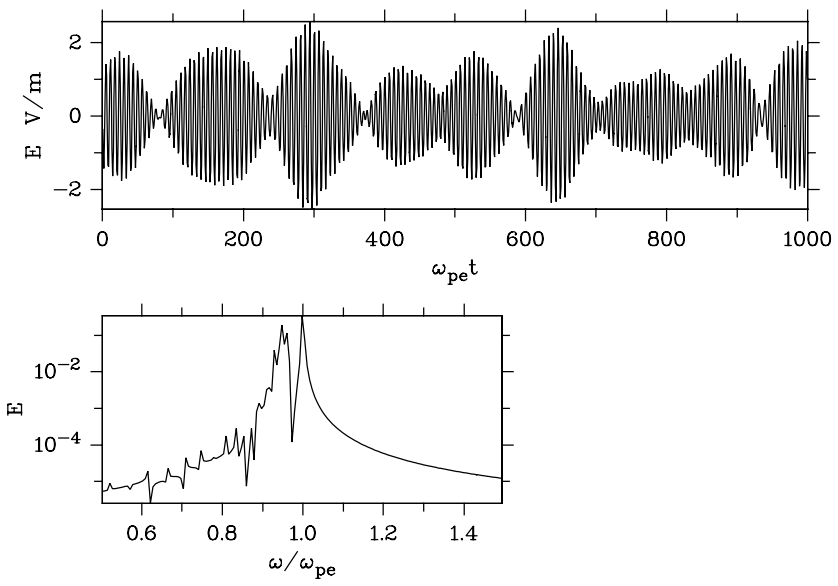

Fig. 1. Influence of beam-aligned plasma oscillations on the Langmuir wave modulation. Temporal representation of the amplitudemodulated field at a fixed position $x / \lambda_{\mathrm{D}}=1000$ (top) and related power spectrum (bottom); box length $3000 \lambda_{\mathrm{D}}$. Beam velocity $V_{\mathrm{b}}=$ $15 v_{\text {the }}$, beam density $n_{\mathrm{b}}=0.05 n_{0}$, amplitude of the plasma oscillation $E_{0}=1.3 \mathrm{~V} \mathrm{~m}^{-1}$. Note the well-developed twin peak with signals at $\omega_{\text {pe }}$ and a value slightly above the frequency of the fastest growing beam-driven mode.
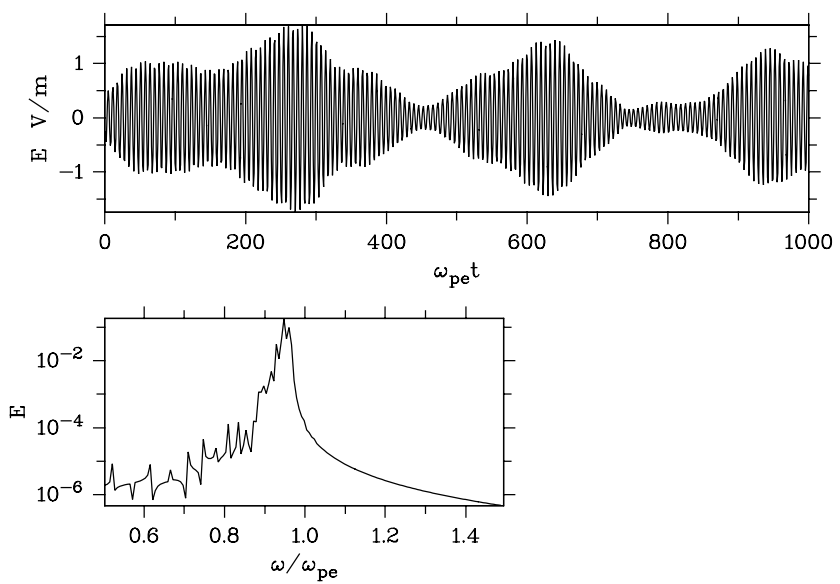

Fig. 2. The same as Fig. 1 but in absence of plasma oscillations. The double peak has disappeared, and the modulation enters an irregular type.

expected, the interference of the plasma oscillations with the instability-generated Langmuir waves results in a more coherent modulation in time representation. The waveform exhibits the beat-type pattern of two modes slightly differing in frequency, indicated by the double peak in the power spectrum.

Although an almost periodic amplitude modulation is clearly reproduced, the separation of the two peaks is smaller than what should be expected. For the parameters used here $\left(n_{\mathrm{b}} / n_{0}=0.05, V_{\mathrm{b}} / v_{\text {the }}=15\right)$, the frequency of the fastest growing mode is $\omega / \omega_{\mathrm{pe}} \approx 0.89$; i.e., the left peak should be downshifted from $\omega_{\mathrm{pe}}$ by $\Delta \omega \approx 0.11 \omega_{\mathrm{pe}}$. The splitting of the

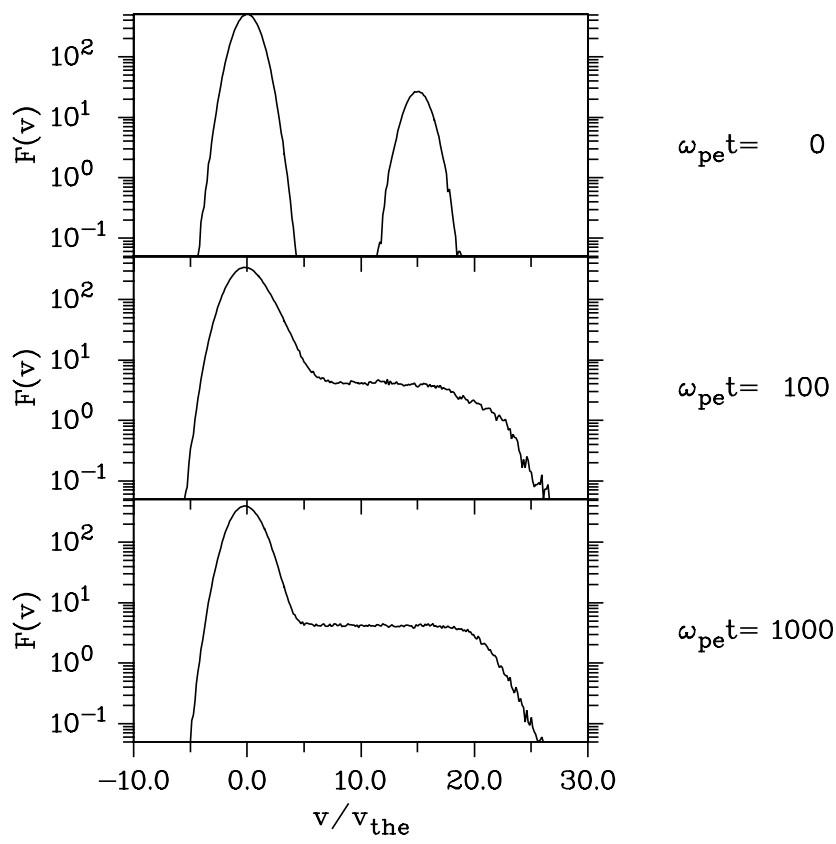

Fig. 3. Time evolution of the electron velocity distribution function, averaged over the simulation box, in a PIC simulation carried out with parameters listed in Table 1.

Table 1. PIC simulation parameters.

\begin{tabular}{ll}
\hline Beam density & $n_{\mathrm{b}}=0.05 n_{\mathrm{o}}$ \\
Beam velocity & $V_{\mathrm{b}}=15 v_{\text {the }}$ \\
Beam temperature & $v_{\text {thb }}=v_{\text {the }}$ \\
Simulation box length & $3000 \lambda_{\mathrm{D}}$ \\
Total number of electrons & 1507902 \\
Time step & $0.025 \omega_{\mathrm{pe}}^{-1}$ \\
Final time & $1000 \omega_{\mathrm{pe}}^{-1}$ \\
Number of grid points & 1024 \\
\hline
\end{tabular}

two peaks in Fig. 1, however, reaches only about half of that value. Thus, in this respect the linear model does not agree with kinetic dispersion theory as well as one might hope.

For comparison, Fig. 2 displays the temporal modulation in the artificial case that plasma oscillations are absent, for otherwise the same parameters. The almost periodic structure has disappeared and is replaced by irregular modulation. Similar results for a lower beam velocity $V_{\mathrm{b}} / v_{\text {the }}=7$ have been shown in Fig. 5 of B1.

\section{PIC simulations}

In this section PIC simulations of the gentle beam-plasma instability are presented for the same beam parameters as used in Fig. 1. The motivation is to test the results of the linear theory by a nonlinear treatment. The simulation parameters are listed in Table 1, based on the natural normalizing 

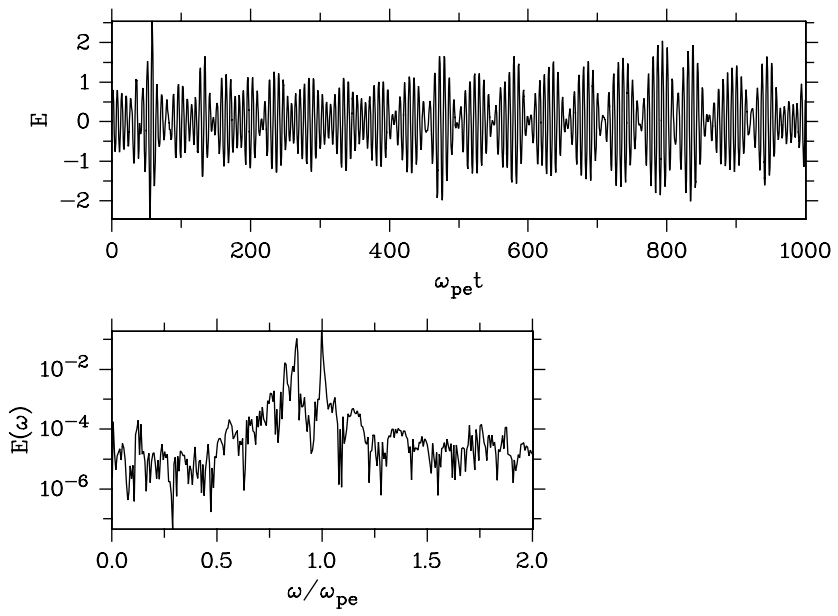

Fig. 4. Temporal representation of Langmuir amplitude modulation as result of a PIC simulation. Beat-type waveform at the position $x / \lambda_{\mathrm{D}}=1000$ (top) and corresponding power spectrum (bottom). The twin peak results from the beating of the fastest growing beamdriven Langmuir mode and plasma oscillations. The latter are seen starting at $t=0$. The minor peak near $\omega / \omega_{\mathrm{pe}} \approx 1.7$ indicates the second harmonic of the dominant wave. The minor peak near $\omega=0$ results from nonlinear interaction of the dominant wave with plasma oscillations.

quantities $n_{0}, \omega_{\mathrm{pe}}, \lambda_{\mathrm{D}}$, and $v_{\text {the }}$. The code does not distinguish between background and beam electrons, and periodic boundary conditions are imposed. The electric field for acceleration of the electrons has been calculated with the help of an implicit momentum method (Langdon, 1985) based on the current Eq. (2). It keeps a tolerable degree of energy conservation. Figure 3 illustrates the evolution of the electron velocity distribution function showing shapes at the start and for two later times. As well known for the gentle beam instability, the beam distribution is rapidly flattened and does not undergo further deformation with growing time. The temporal amplitude modulation is illustrated in Fig. 4. It displays the field variation with time at the position $x / \lambda_{\mathrm{D}}=1000$ and the associated power spectrum. The field is here in units of $m_{\mathrm{e}} / e v_{\text {the }} \omega_{\text {pe }}$. In the reference plasma it corresponds to $\approx 1.8 \mathrm{~V} \mathrm{~m}^{-1}$; i.e., the field is on average two times stronger than predicted by linear theory (Fig. 1). The time variation of the longitudinal field exhibits clearly the structure of a regular string of wave packets. The scenario shown in Fig. 4 is consistent with the beating of plasma oscillations and the dominant mode of the instability, the frequency of which is indicated by the left peak in the power spectrum. It exhibits a downshift of $\Delta \omega \approx 0.13 \omega_{\text {pe }}$ in better agreement with kinetic dispersion theory. Note the plasma oscillation released by the finite initial current, seen at the very beginning of the field evolution, prior to the onset of the instability.

The second run has more the character of a numerical experiment and starts from an artificial current-free state. This is achieved by a slight drift of the background electrons
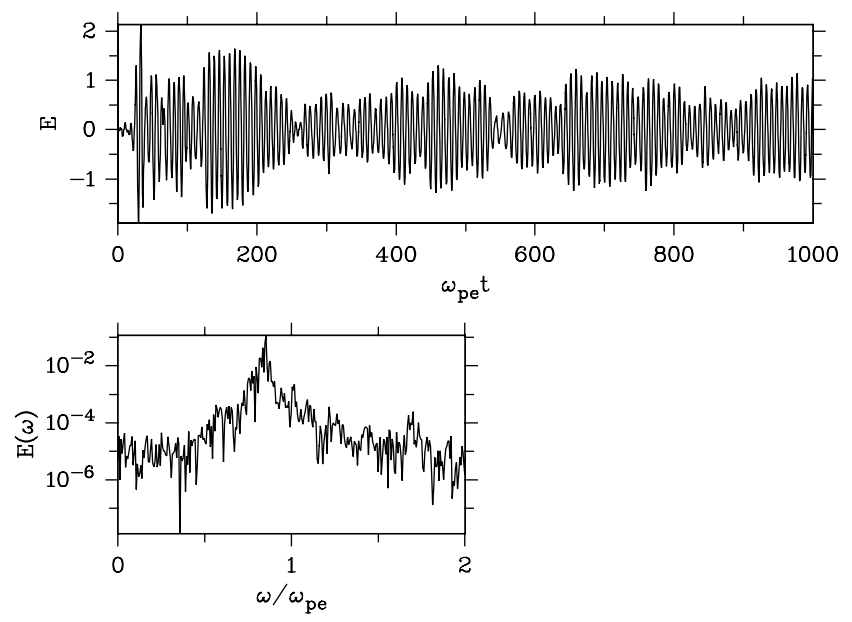

Fig. 5. The same as Fig. 4, but with a current-free state at the beginning of the simulation, arranged by a small drift of the background electrons in negative direction. The modulation renders chaotic by the absence of the initial current.
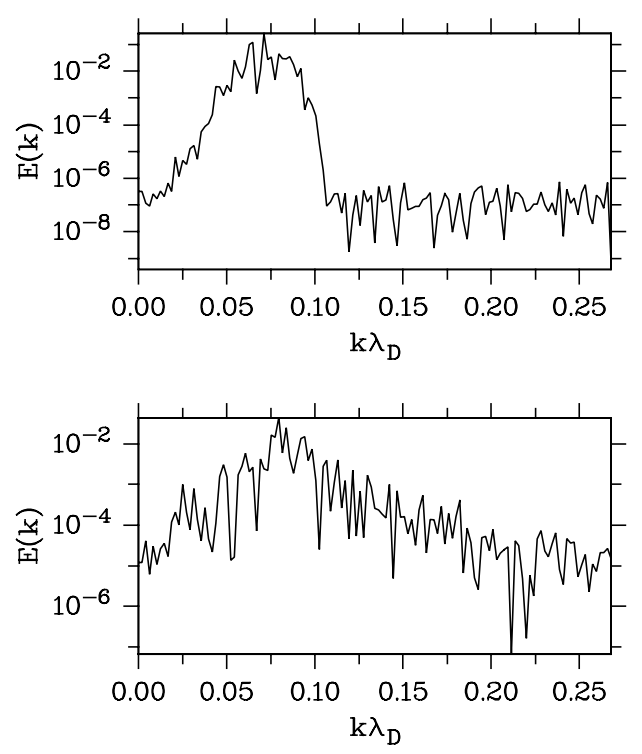

Fig. 6. Comparison between spatial power spectra of beamgenerated Langmuir waves in the linear model (top) and in the PIC simulation (bottom). Both spectra peak near $k \lambda_{\mathrm{D}} \approx 0.075$, the wave number of the fastest growing mode.

against the beam direction such that $n_{\mathrm{e}} v_{\mathrm{e}}(0)+n_{\mathrm{b}} V_{\mathrm{b}}=0$. The results are displayed in Fig. 5. In comparison to Fig. 2 the coherent modulation has disappeared and the variation of the field envelope comes about only by the superposition of the broad range of unstable waves excited by the instability, as described in B1. The resulting modulation pattern enters an irregular structure.

Although the focus in our analysis is on temporal modulation, we compare in Fig. 6 the power spectrum of spatial field profiles obtained in the linear model and in PIC 
simulations. After the growth period they change little with time. In both cases the spectrum peaks near $k \lambda_{\mathrm{D}} \approx 0.075$, the wave number of the dominating unstable Langmuir wave for the beam parameters used here. Differences appear in the fall-off with growing wave number. While the linear model predicts a steep decrease, the PIC simulation produces a mitigated slow-down. This may indicate that unstable shorter wavelength modes reach higher saturated amplitudes than allowed in the linear model.

The results of linear theory and PIC simulations show qualitative similarity. The lack of quantitative agreement, especially for the period of the modulation and the field amplitude, can possibly be ascribed to the serious simplifications made in the linear model for the processes occurring during the growth phase of the instability. One of them is the assumption of an abrupt change of the beam distribution to a plateau, which forces a common saturation time for all growing modes regardless of their wave number. Nevertheless, we may conclude that the PIC simulations support the linear analysis with respect to both the random and the regular appearance of the amplitude modulation.

An inspection of the two most pronounced double peaks observed by Hospodarsky et al. (1994) (their Fig. 5) and Sigsbee et al. (2010) (their Fig. 3d) reveals peak separations of $\Delta \omega=0.13 \omega_{\mathrm{pe}}$ and $\Delta \omega=0.08 \omega_{\mathrm{pe}}$, respectively. Corresponding beam parameters are not well known, but these values fit into the theoretical picture developed above for dense electron beams with $n_{\mathrm{b}} / n_{0} \geq 0.05$.

\section{Summary}

Langmuir waveforms observed in space plasmas show a considerable variety. Most of them exhibit an irregular structure, consisting of wave periods of variable length, and exhibit a single peak near $\omega_{\text {pe }}$ in the power spectrum. Occasionally the waveform is almost coherently modulated and has the form of a string of wave packets with nearly the same time scale. In this report a linear model to describe irregular Langmuir wave modulation, developed in a previous paper (B1), is completed to include periodic modulation as well. This is achieved by a combination of the random modulation mechanism and beam-aligned plasma oscillations. These oscillations appear simultaneously with the beam, prior to the onset of the instability, presupposed the interaction region keeps a sufficient degree of homogeneity. In cases when the amplitudes of plasma oscillations and the fastest growing beamdriven mode are comparable, a beat-type waveform may develop where two modes of slightly different frequency interfere with each other. This results in two distinct signals (double peak) in the power spectrum. The two peaks are well separated, however, only for beams with a relative density of at least a few percent.

In order to check the linear model by a nonlinear code, PIC simulations have been carried out with the same parameters.
The results support the predictions of the linear approach, in particular the occurrence of beam-aligned plasma oscillations.

The fact that no indications of this simple phenomenon have been seen in former simulations of the gentle beamplasma instability may have the reason that in general lower beam densities were in the focus or an artificial current-free plasma was preferred to start the simulation. In both cases coherent Langmuir amplitude modulation does not occur and double peaks are absent in the power spectrum.

We may conclude that the linear dispersion model accounts for basic features of the Langmuir modulation, including both the irregular type and the formation of coherent structures in which wave packets repeat their shape almost periodically. This gives evidence that the modulation of Langmuir waves occurs primarily without nonlinear mechanisms and thus can be seen with weak electric fields.

Acknowledgements. The author would like to express his thanks to the two referees for their constructive criticism of the manuscript.

Topical Editor I. A. Daglis thanks S. C. Buchert and one anonymous referee for their help in evaluating this paper.

\section{References}

Baumgärtel, K.: A linear model for amplitude modulation of Langmuir waves in weak electron-beam plasma interaction, Ann. Geophys., 31, 31-38, doi:10.5194/angeo-31-31-2013, 2013.

Daldorff, L. K. S., Pecseli, H. L., Trulsen, J. K., Ulriksen, M. I., Eliasson, B., and Stenflo, L.: Nonlinear beam generated plasma waves as a source for enhanced plasma and ion acoustic lines, Phys. Plasmas, 18, 052107, doi:10.1063/1.3582084, 2011.

Gurnett, D. A., Hospodarsky, G. B., Kurth, W. S., Williams, D. J., and Bolton, S. J.: Fine structure of Langmuir waves produced by a solar electron event, J. Geophys. Res., 98, 5631-5637, 1993.

Hospodarsky, G. R., Gurnett, D. A., Kurth, S. W., Kivelson, M. G., Strangeway, R. J., and Bolton, S. J.: Fine structure of Langmuir waves observed upstream of the bow shock at Venus, J. Geophys. Res., 99, 13363-13371, 1994.

Kellog, P. I., Morison, S. J., Goetz, K., Howard, R., Bougeret, J. L., and Kaiser, M. L.: Early wind observations of bow shock and foreshock waves, Geophys. Res. Lett., 23, 1243-1246, 1996.

Kojima, H., Furuya, H., Usui, H., and Matsumoto, H.: Modulated electron plasma waves observed in the tail lobe: Geotail waveform observations, Geophys. Res. Lett., 24, 3049-3052, 1997.

Langdon, A. B.: Implicit plasma simulations, Space Sci. Rev., 42, 67-83, 1985.

Matsukiyo, S., Treumann, R. A., and Scholer, M.: Coherent waveforms in the auroral upward current region, J. Geophys. Res., 109, A06212, doi:10.1029/2004JA010477, 2004.

Muschietti, L., Roth, I., and Ergun, R. E.: On the formation of wave packets in planetary foreshocks, J. Geophys. Res., 101, 1560515613, 1996.

Omura, Y., Matsumoto, H., Miyake, T., and Kojima, H.: Electron beam instabilities as generation mechanism of electrostatic solitary waves in the magnetotail, J. Geophys. Res., 101, 2685-2697, 1996. 
Pottelette, R., Ergun, R. E., Treumann, R. A., Berthomier, M., Carlson, C. W., McFadden, J. P., and Roth, I.: Modulated electronacoustic waves in auroral density cavities: Fast observations, Geophys. Res. Lett., 26, 2629-2632, 1999.

Sauer, K. and Sydora, R.: Mode crossing effects at electron beamplasma interaction and related phenomena, Plasma Phys. Control. Fusion, 54, 124054, doi:10.1088/0741-3335/54/12/124045, 2012.

Sigsbee, K., Kletzing, C. A., Pickett, J. S., Gurnett, D. A., Schwartz, S. J., Levebvre, B., Lucek, E., Fazakerley, A. N., and Kucharek, H.: Characteristics of Langmuir electric field waveforms and power spectra exhibiting nonlinear behavior in Earth's foreshock, J. Geophys. Res., 115, A10251, doi:10.1029/2009JA014948, 2010.
Silin, I., Sydora, R., and Sauer, K.: Electron beam-plasma interaction: Linear theory and Vlasov-Poisson simulations, Phys. Plasmas, 14, 012106, doi:10.1063/1.2430518, 2007.

Umeda, T.: Vlasov simulation of amplitude modulated Langmuir waves, Phys. Plasmas, 13, 092304, doi:10.1063/1.2348088, 2006.

Usui, H., Furuya H., Kojima, H., Matsumoto, H., and Omura, Y.: Computer experiments of amplitude-modulated Langmuir waves: Application to Geotail observation, J. Geophys. Res., 110, A06203, doi:10.1029/2004JA010703, 2005. 